\title{
Web Based Intelligent Decision Support System for Type 2 Diabetes Patients
}

\section{F. Sahar}

\begin{abstract}
Intensive treatment of type 2 diabetes can respond as a considerable decrease in the complications related to diabetes. In spite of specialist care there are still many patients that have poor control over their diabetes and the chronic nature of type 2 diabetes makes patients dependent on their care providers. In Type 2 diabetes patients require empowerment and self-management for initial and long term treatment. The required transfer of care from health care providers to patients needs an intervention of computerized decision support systems. In widespread health care practice the interventions that have demonstrated effective results in the management of chronic and/or progressive diseases have often been difficult to develop because of disease nature. The paper describes a web based intelligent decision support system (IDSS) for type 2 diabetes patients, where more emphasis is put on patient empowerment and self-management in treatment. The IDSS is based on well documented decision support system, designed mainly for type 2 diabetes patients. The scope of IDSS is broad from being mainly used by type 2 diabetes patients as a tool for their empowerment, self-management, communication, and education.
\end{abstract}

Index Terms-Exercise, IDSS, selfmanagement, type 2 diabetes, web application

\section{INTRODUCTION}

According to the Diabetes Control and Complications Trial Research Group, in both developed and developing countries diabetes as a major chronic disease is a growing public health problem [1]. In 2007 International Diabetes Federation (IDF) published standardized global estimates for the prevalence of diabetes. According to IDF, worldwide diabetes currently affects 246 million people and is expected to affect 380 million by 2025 . IDF statistical prediction identifies that every year due to diabetes 3.8 million people die and 7 million develop diabetes. The most common types of diabetes are Type 1 and Type 2 [2].

According to IDF, in developed countries there are 85 to $95 \%$ people are suffering from type 2 diabetes. In type 2 diabetes, a person is not dependent on exogenous insulin and are not ketosis-prone, but hyperglycaemia control may require insulin when it's not realize with diet alone or with oral hypoglycaemic agents [2]. P. Zhang writes that, in elder type-2 diabetes is becoming more common due to obesity, lack of exercise, family history of diabetes and heart diseases [3]. The type-2 diabetes is either progressive or chronic in nature and this makes patients dependent on their care providers such as family and the local health services [3]. However, a main challenge is to provide necessary support

Manuscript received January 24, 2013; revised April 5, 2013.

The author is with Tampere University of Technology, Finland (e-mail: farrukhsahar@yahoo.com). and empowerment to diabetes patients at initial stages and over the long term, that will allow their self care management in a feasible and cost effective ways [4].

The considerable loss in quality of life associated with the disease is mainly due to the late complications such as kidney failure, blindness, and circulatory diseases etc. Although research has proved that intensive diabetes treatment can respond as a substantial reduction in complications but using conventional means this can be difficult to achieve. Even though studies have proved that the implementation of decision support systems may provide support to solve some of the problems. Studies explained that during the last two decades until now none of the developed systems gained widespread popularity or acceptance [5]. One reason for this low acceptance rate might be the problems in handling the intrapatient and interpatient variability and some other reasons might be the variations in the data involved such as fever, stress, diet, and exercise etc.[6]. Previous studies identified that the decision support system in the classical sense is not enough and for the successful implementation of decision support systems patient empowerment should be taken into consideration. The empowerment strategy might be learning, education and communication [7].

\section{BACKGROUND}

Diabetes type 2 is increasingly common, mainly because of increase in obesity and inactive lifestyle. The type 2 diabetes can be prevented by interventions that affect the lifestyles of patients such as diet and exercise [8]. In many fields decision support is a broadly applicable concept that supports many activities in human life in different contexts. In health care domain the computerized clinical decision support systems are use to assist healthcare staff by supporting them to complete their work more accurately and efficiently and it also improves the quality of patient care. In many situations DSS facilitate appropriate treatment of choice such as medicine duration and dosage. In many cases the DSS can be either work independent or dependent on other Hospital information systems.

For many years Physicians have used these decision support tools to bring the point of care (point of decision making) as aids. These tools include antibiotic therapy guides, pocket editions of texts, treatment synopses and diagnostic algorithms. Therefore decision support tools are not new. At present there are many computerized decision support tools. Computerized decision support tools are helpful in the standardization of clinical decision making and lead to favorable impacts on patient outcome [9]. These tools include care supporting applications with hand-held devices or stationary that provides self-management. Another DSS tools 
is electronic patient records (EPR) that is use to capture, store the patient data, provides identification of temporal changes, consistency, and reproducibility. To provide patient specific data the computerized protocols are use to drive patient data, thus preserving individualized care while treatment. In addition decision support systems and computer information systems that are currently being implemented in medicine have offered improved outcomes [10]. According to the studies the computerized decision support systems can provide considerable benefits in the process of prescribing medication, which is the most common intervention in medicine [11]. This result in an improved dosing, improved anticoagulation therapy, reduced adverse drug events, and improved antibiotic use [10].

\section{A. The Role of Physical Activity in Type 2 Diabetes}

The elderly population, increase in obesity, and a more sedentary population, are the keys factors behind the high prevalence rate of type 2 diabetes. People lifestyle behavioral interventions, such as physical activity, can play a vital role to prevent or delay the onset of type 2 diabetes for those at risk [12]. In addition for type 2 diabetes patients, physical activity has considerable positive effects such as it may help to improve glycemic control, positively affect other coronary heart disease risk factors as well as reduce blood pressure [13]. In spite of the known significance of exercise regarding physical and mental health, Most of the people with selfreported diabetes fail to perform required amount of physical activity [14]. As a result it remains a challenge for health care practitioners and researchers to promote physical activity among type 2 diabetes patients [15].

In studies, most people believed that physical activity had some benefit to their physical and/or mental health. For example, many people experienced positive effects specific to their diabetes condition and believe that physical activity helps to regulate blood sugar levels and maintain weight [15]. According to studies most patients felt they need sufficient skills and knowledge to carry out regular physical activity. Reading articles, web sites, and books are the most common source of acquiring skill and knowledge. Other sources are family doctor advices, diabetes seminars and general lifelong learning. Furthermore, according to some patient views they learned by observing others, specifically personal trainer/ instructor, TV/videos, and peers/ family [15].

\section{B. The Role of Nutrition Therapy in Type 2 Diabetes}

Determining the nutritional needs for a specific patient with diabetes, writing a diet order according to their specific needs, and finally combining the current nutrition principles and recommendations together can be a difficult task. Studies shows that in hospital diets are most of the time ordered by calorie levels based on the "ADA diet," [16]. In conclusion research studiesexplained that patient nutrition needs often differ in the home settings as compared to hospital setting. For example the diabetes treatment plan used in the home may differ from hospital. In studies it is observed that dietitians who specialize in nutrition can play a significance role in the health care management of diabetes patients. In studies it is recommended that a registered dietitian with knowledge and skills in medical nutrition therapy can serve as the team member to provide medical nutrition therapy. The responsibility of dietitian includes integrating information about the patient's clinical history, lifestyle and, eating habits in order to establish treatment goals for patient [17].

\section{RELATED WORK}

Many studies have identified that computerized decision support systems can be successfully implemented in many other areas of health care domain. These areas include electronic documentation and information retrieval [18], physician order entry and adverse drug events [19],[20] as well as preventive care and follow-up [21], [22].

\section{A. CompTMAP}

In Texas among "real-world" clinical settings, the DSS has been developed, tested, and used for depression treatment [10]. For the treatment of major depressive disorder they designed and developed a computerized decision support system (CompTMAP), the main task of CompTMAP is to provide support in diagnosis, treatment, follow-up, as well as in preventive care. The CompTMAP based on evidence-based guidelines and can be incorporated into the clinical setting [10]. In surveys physicians have indicated a positive response towards CompTMAP [10]. By taking the advances in automation CompTMAP provides more complete clinical support to physicians in clinical practice. [10]. CompTMAP provides decision support for both care taker and care provider. It supports patients in diagnosis, appropriate treatment options, and follow-up as well as preventive care. Moreover it supports physicians in order entry, information retrieval, alert systems, and electronic documentation [10].

\section{B. The DiasNet}

The DiasNet system is basically designed for clinicians to give advices on insulin and it is also used by patients as tool for communication and education. Patients can make use of their own data, to adjust insulin doses or meal sizes. In this way patient can try different therapeutic and dietary alternatives, this will help the patient to achieve glycaemic control [7].

\section{Consistent Carbohydrate Diabetes Meal-planning System}

To provide up-to-date food service to patients in health care organizations "the consistent carbohydrate diabetes meal-planning system" was developed. The system is based on the amount of carbohydrate offered at each meal. In the system amount is consistent from meal to meal and day to day. The selection of meals are based on diet principles saturated cholesterol and fats are limited, and protein content falls within $15-20 \%$ of calories as usual diet's content. The focus is on the total amount of carbohydrate contained in the meal in spite of the type of carbohydrate in meal served [23].

\section{Successful Studies on Type 2 Diabetes Patients}

To test the feasibility of long-term intervention with the focus on life-style changes a selection is made from a previously reported 5-year screening program of 6,956 47-49-year-old Malmo males. In study a series of 181 subjects with impaired glucose tolerance and 41 subjects with early-stage Type 2 diabetes mellitus were investigated. The 
study concluded that long-term intervention in the form of diet and physical exercise is feasible even on a large scale and considerable health improvement can be accomplished which may contribute to postpone or prevent manifest diabetes [24].

Another investigated study based on documented 1419 incident cases of type 2 diabetes proved that physical activity level is associated with substantial decrease in risk of type 2 diabetes. The incident cases were documented during 8 years of follow-ups $(534,928$ person-years) [25].

\section{Proposed InTELligent Decision Support SySTEM}

The core functionality of proposed intelligent decision support system (IDSS) is related to diet and exercise models. Each model is associated with two variables one variable is height and another variable is weight. Both of these variables are used to calculate Body Mass index (BMI) of user. The values of both of these variables give an ability to IDSS to handle the uncertainty. The IDSS is operated in two modes, the learning and prediction mode. In the learning mode the data on weight and height are used to estimate the patient specific parameter. In the way the system switch itself according to specific patient. In the prediction mode the system uses the same data on height and weight to make prediction about the routine exercises.

\section{IDSS FUNCTIONALITY}

Based on the experience from previous Integrated Mobile Information System (IMIS), a new web based system, IDSS has been developed. The scope of this web based application covers type 2 diabetes patients as well as clinicians. Diabetes type 2 patients will use IDSS for their self-management regarding their diet and exercise and clinicians will use IDSS to give advises on diet and exercise. In the patient perspective IDSS behave as a tool for education and communication. The important part in the education of diabetes patient is learning how to attain achieve glycaemic. Another aspect of the IDSS is the learning environment. Patients can experiment with their own data adjusting diet and/or exercises routines and learn how to deal with different health conditions. The IDSS also educate patients that how to achieve glycaemic control. In this way different dietary and exercise alternatives can be taken into consideration without taking the risk of actually experiencing hyperglyceamic or hypoglyceamic situations. While experiencing the IDSS patients can learn about the effect calories, exercises routine and carbohydrate intakes. For example patient can learn about the effect of increased exercise in summer vocations. Furthermore as comparing to other traditional diabetes educational materials the major benefit of IDSS is, it utilizes the patient's own data for diabetes management. In addition the system gives empowerment to patient and enables new ways of self-treatment.

Patient can use IDSS web application on PC at any place where internet connection is available so even sitting at home patient can use IDSS for their diabetes management. In case of problem or questions s/he can email or phone to clinicians who can use the IDSS in his office PC to give suggestion on diet and exercise. The IDSS provides a clear overview of diet and exercise routines to patients instead of patient spend their most of the time in managing and updating a hand written diary. To demonstrate the usage of IDSS, a particular patient scenario will be presented in which different system functionalities will be identified. In order to increase the acceptance rate of IDSS among patients as a tool for their self-management strong consideration has been put on Graphical user Interface (GUI) to develop a simple and user-friendly interface. There are four main parts of IDSS GUI, in first a "data section" to enter Id, password, weight and height, a "future section" to experiment different dietary and exercise alternatives, a "history section" to help the patients in tracking the changes that he/she has made when experimenting with data, and a "suggestion section" to graphically display the data regarding suggested exercise routines for every specific patient.

\section{A. Data Section}

As the first step when the patient enters in the IDSS, the Id and password needs to be entered. After this user can select any option from available options of Meal planner" and" Activities". At last "height" and" weight" have to be entered. This is done via the data section which besides providing functionality for entering the user data also displays patient information. Such as on "Meal planners" page its displays weight, height, daily calories, meal, available calories, calories used, remaining calories, and meal selection history (Meal name, serving, food name, calories).

Furthermore on activities page, data section displays patient information such as weight, height, calories lost, total calories lost, suggested activities and patient activities (activity id, activity name, loose calories).

In overall IDSS design simplicity is taken into consideration and to enter the data in IDSS data section following routine of steps is suggested.
1) Enter the ID and password on IMIS log-in.
2) Click on "different tools" and select "diabetic tools".
3) Click on "IDSS log-in".
4) Enter the "user name" and "password" and press "submit" button.
5) By clicking select either "meal planner" or "activities".
6) Click on "height" field, enter the height and click on "change" link.
7) Click on "weight" field, enter the weight and click on "change" link.

\section{B. Future Section}

After entering the "height" and "weight" in either "meal planners" or "activities" page, the user is able to enter in the future section for further experiments. On "meal planners" page the user can experiment with different dietary alternatives. On the other hand on "activities" page the user can experiment with different exercise alternatives. In the future section of "meal planners" page, "meal", "items", and "servings" can be changed by simply using the mouse to select the item in the "drop down list". After selecting the meal, items, and servings user can click on "enter link" and immediately IDSS will show the resulting calories prediction.

In addition in the future section of "activities" page, activities can be changed in a same manner with "drop down 
list" as in a future section of "meal planners" page. After selecting the "activities" the "hours" and/or "minutes" have to be entered. When the user press the "enter button", the IDSS will immediately show the resulting "calories lost" and "total calories lost" predictions. For example Fig. 1 illustrates the data on "meal planners" page, where the IDSS predicted "daily calories" and "available calories, "calories used", "calories remaining", and "calories according to specific food items" are displayed. Furthermore Fig. 2 illustrates the data on "activities" page, where the IDSS predicted "calories lost" and" total calories lost" are displayed.

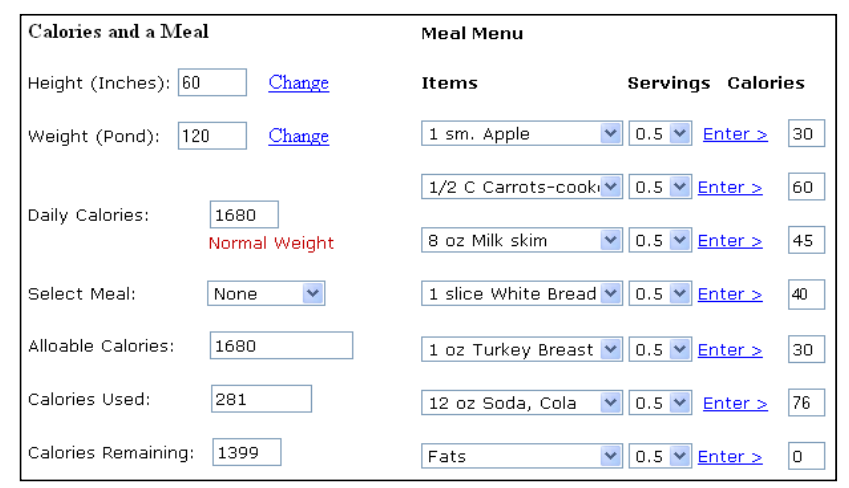

Fig.1. illustrates the data on "meal planners" page

Daily Activities
Activities: Cycling $\quad v$ Hours: $0 \quad$ Minutes: 0 Enter
Calories Lost: 480
Total Calories Lost: 1680

Fig. 2. illustrates the data on "activities" page

\section{History Section}

After entering the "height" and "weight" in the data section and experimenting with different dietary and/or exercise alternatives all entries will be saved in the history section. The history section helps the patients to track the changes when experimenting with data. For example Fig. 3 illustrates the history of "meal planners" page about "id", "meal name", "servings", "food name", and "calories". Moreover Fig. 4 illustrates the history of "activities" page about "activity id", "activity name", and "loose calories".

\begin{tabular}{|c|c|c|c|c|c|}
\hline \multicolumn{6}{|c|}{ Breakfast } \\
\hline \multicolumn{6}{|c|}{ ID Meal Name Servings Food Name Calories } \\
\hline & Delete 349 & 9 Breakfast & 0.5 & $1 \mathrm{sm}$. Apple & 30 \\
\hline
\end{tabular}

Fig. 3. illustrates the history of "meal section"

\begin{tabular}{|llll|}
\hline \multicolumn{2}{|c|}{ Patient Activities } & \\
& Activity ID & Activity Name & Loose Calories \\
\hline$\underline{\text { Delete }}$ & 97 & Walking Level at 1mph & 50 \\
$\underline{\text { Delete }}$ & 98 & Jogging & 540 \\
Delete & 99 & Walking Level at 1mph & 120 \\
Delete & 100 & Jogging & 540 \\
\hline
\end{tabular}

Fig. 4. illustrates the history of previous "activities"

\section{Suggestion Section}

When the user has entered "height" and" weight" on "activities" page, then the system will suggest the user how much calories s/he can lose by exercise according to entered "height" and "weight". For example Fig. 5 illustrates the system predicted weight status "Normal Weight" and also suggestions for user "Note: you need to lose 1000 calories in one week". On "activities" page IDSS also inform user when the user is crossing the suggested calories loose limit for exercise. For example Fig. 6 illustrates the system suggestion "Note: You are crossing (Walking Level at $1 \mathrm{mph}$ ) suggested calories loose limit." when the user has crossed the suggested calories loose limit for exercise. After the completion of data section and future section, on the "activities" page the IDSS dares to suggest more alternatives for exercise routines. The suggestion section helps the patient to aware about other exercise routines alternatives. The important aspect is that the IDSS suggests the exercise routine according to the particular patient needs. For example Fig. 7 illustrates the suggested activities alternatives.

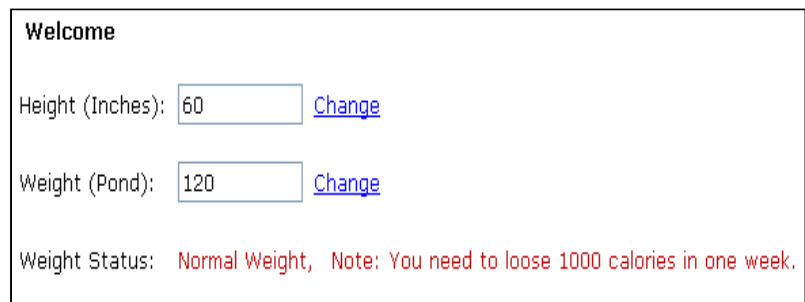

Fig. 5. illustrates the IDSS suggestions for user

Daily Activities
Activities: Walking Level at $1 \mathrm{mph} \vee$ Hours: $0 \quad$ Minutes: 0
Calories Lost: 240
Total Calories Lost: 360
Note: You are crossing (Walking Level at 1mph) suggested calories loose limit.
$\quad$ Follow the Suggested Activities.
Patient Activities Save

Fig. 6. illustrates suggested calories loose limit scenario.

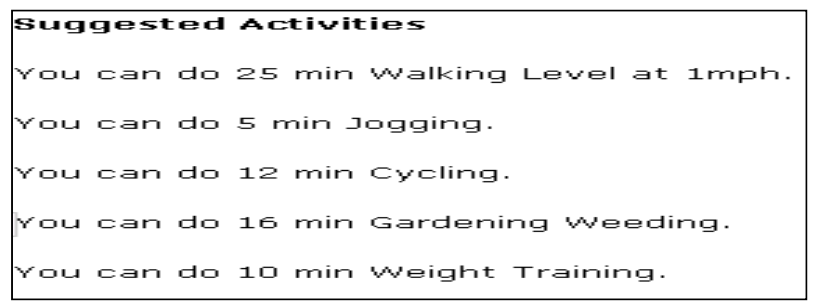

Fig. 7. illustrates the suggested activities alternatives

\section{DISCUSSION}

The type 2 diabetes can be prevented by interventions that affect the lifestyles of patients such as diet and exercise [8].In health care domain Decision support systems areuse to complete health care activities more accurately and efficiently and it improves the quality of patient care. Research study [24] has proved that decrease in the prevalence of diabetes is directly associated with changes in lifestyle. Research studies also concluded that exercise and/or diet are the main interventions that may lead to a considerable decrease in the incidence of diabetes. The 
proposed IDSS can facilitate appropriate treatment of type 2 diabetes patients by giving them empowerment and self-management for initial and long term treatment. The important aspect is that the IDSS always respond according to the particular patient profile. Patient can achieve glycaemic control using IDSS and s/he can learn how to manage diabetes by dealing with their own data. With the support of IDSS the quality of patient's life style will increase when the patient is enable to adjust his/her meal sizes and exercise routines according to their diabetes treatment plan. The IDSS sections of data, future, history, and suggestion can play a big role to support patient in the management of data, time, and history.

\section{CONCLUSION}

In general it is assumes that advance computer systems capable of supporting patient-specific management can improve the health care of data intensive and complex disease such as type 2 diabetes, but different research results identified that only limited evidence support this assumption. According to the increasing prevalence of type 2 diabetes the improvement in type 2 diabetes care is a big challenge. Researchers are still trying to transfer the applicable treatments and evidences into effective care for type 2 diabetes patients using computerized decision support systems. On the other side in provider's perspective, the complexity and large number of diabetes treatment goals, as well as screening and analyzing requirements represents the core barriers to deliver a patient-specific high quality care.

In this study, we demonstrated that web-based, patient-specific, intelligent decision support system based on meal size and exercise routines. The meal size and exercise routines that are core factors in the treatment of diabetes type 2 patients. If we provide treatment to type 2 diabetes patients according to the treatment significance of these two factors then we can get considerable benefits regarding glycaemic control. Our study has several limitations at this stage it is quite difficult to cover all the dietary food items and also their accurate amount of Nutrition. So, IDSS predicted values are somehow near to accurate but not $100 \%$ accurate.

\section{REFERENCES}

[1] The Diabetes Control and Complications Trial Research Group, "The effect of intensive treatment of diabetes on the developme430nt and progression of long-term complications in insulin-dependent diabetes mellitus," The New England Journal of Medicine, vol. 329 (1993), pp. 977-986.

[2] International Diabetes Federation, Diabetes Facts and Figures, Brussels: Belgium, 2009.

[3] P. Zhang, "Multi-agent systems supported collaboration in diabetes healthcare," Blekinge Tekniska Högskola, Sweden, 2008.

[4] H. Garth McKay et al., "Internet-Based Diabetes Self-Management and Support: Initial Outcomes from the Diabetes Network Project," Rehabilitation Psychology, vol. 47, no. 1, pp. 31-48, 2002.

[5] U. Bott, S. Bott, D. Hemmann, and M. Berger, "Evaluation of a holistic treatment and teaching programme for patients with Type 1 diabetes who failed to achieve their therapeutic goals under intensified insulin therapy," Diabetes Med, vol. 17, pp. 635-643, 2000.

[6] J. Wyatt, C. Friedman, and D. Spiegelhalter, "Evaluating medical decision-aids," in State-of-the-Art and Future Directions in AI," in Medicine, S. Uckun (Ed.), Elsevier, Amsterdam, 1994.
[7] S. Plougmann et al., "DiasNet - a diabetes advisory system for communication and education via the internet," Department of Medical Informatics and Image Analysis, Aalborg Uniersity, Fredrik Bajersej 7 D1, DK-9220, Aalborg, Denmark, International Journal of Medical Informatics, vol. 64, pp. 319-330, 2001.

[8] S. Berman, "The AMA clinical quality improvement forum on addressing patient safety," Jt Comm J Qual Improv, vol. 26, pp. 428-33, 2000.

[9] A H Morris, "Decision support and safety of clinical environments," Qual. Saf. Health Care, 2002, vol. 11, pp. 69-75, doi:10.1136/qhc.11.1.69.

[10] M. H. Trivedi et al., "A Computerized Clinical Decision Support System as a Means of Implementing Depression Guidelines," Psychiatric Services, vol. 55, no. 8, August 2004.

[11] D. C. Classen, S. L. Pestotnik, S. Evans, et al., "Adverse drug events in hospital patients," JAMA, vol. 279, pp. 301-306, 1997.

[12] J. Tuomilehto, J. Lindstrom, J. G. Eriksson, et al, "Prevention of type 2 diabetes mellitus by changes in lifestyle among subjects with impaired glucose tolerance," N Engl J Med, vol. 344, pp. 1343-1350, 2001.

[13] N. G. Boule, G. P. Kenny, E. Haddad, G. A. Wells, R. J. Sigal, "Metaanalysis of the effect of structured exercise training on cardiorespiratory fitness in type 2 diabetes mellitus," Diabetologia, 2003, vol. 46, pp. 1071-1081.

[14] Health Canada, Diabetes in Canada, 2nd ed. Ottawa: Health Canada; 2002.

[15] J. E. Barrett et al., "Physical activity and type 2 diabetes exploring the role of gender and income," The Diabetes Educator, 2007, vol. 33, pp. 128, DOI: $10.1177 / 0145721706297453$.

[16] American Diabetes Association, "Translation of the diabetes nutrition recommendations for health care institutions," Diabetes Care, vol. 20, pp. 106-108, 1997.

[17] R. G. Schafer, B. Bohannon, M. Franz, J. Freeman, A. Holmes, S. McLaughlin, L. B. Haas, D. F. Kruger, R. A. Lorenz, and M. M. McMahon, "Translation of the diabetes nutrition recommendations for health care institutions," Diabetes Care, vol. 20, pp. 96-105, 1997.

[18] M. H. Trivedi, J. K. Kern, and S. M. Baker, et al., "Computerized medication algorithms and decision support systems in major psychiatric disorders," Journal of Psychiatric Practice, vol. 6, pp. 237-246, 2000.

[19] D. W. Bates, L. L. Leape, D. J. Cullen et al., "Effect of computerized physician order entry on prevention of serious medication errors," JAMA, vol. 280, pp.1311-1316, 1998.

[20] L. T. Kohn, J. M. Corrigan, and M. S. Donaldson. (November, 1999). To err is human: building a safer health system. Committee on Quality Health Care in America, Institute of Medicine. [Online]. Available at http://books.nap.edu/html/to_err_is_human/exec_summ.html.

[21] M. E. Johnston, K. B. Langston, R. B. Haynes, et al., "Effects of computer-based decision support systems on clinician performance and patient outcome," Annals of Internal Medicine, vol. 120, pp.135-142, 1994.

[22] D. K. Litzelman, R. S. Dittas, M. E. Miller, et al., "Requiring physicians to respond to computerized reminders improves their adherence with preventative care protocols," Journal of Internal Medicine, pp. 311-317, 1993.

[23] American Diabetes Association, "Evidence- based nutrition principles and recommendations for the treatment and prevention of diabetes and related complications (Position Statement)," Diabetes Care, vol. 26 (Suppl. 1), pp. S51-S61, 2003.

[24] K.-F. Eriksson and E. Lindgiirde, "Prevention of Type 2 (non-insulin-dependent) diabetes mellitus by diet and physical exercise, The 6-year Malmo feasibility study," Department of Internal Medicine, Section of Preventive Medicine, Malta6 General Hospital, University of Lund, Malm6, Sweden, Diabetologia, vol. 34, pp. 891-898, 1991.

[25] F. B. Hu, et al., "Walking compared with vigorous physical activity and risk of type 2 diabetes in women a prospective study," JAMA, vol. 282, pp. 1433-1439, 1999.

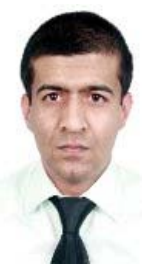

Farrukh Sahar was born in Quetta, Pakistan in 1980 He got his M.S.degree in Computer Science from Blekinge Tekniska Högskola, Sweden. He is a PhD Student at Tampere University of Technology, Finland.

His Research interests are Remote methods for long-term UX evaluation, Long-term UX evaluation of multi-component products. 